\title{
How important is personal exposure assessment in the epidemiology of air pollutants?
}

\section{J W Cherrie}

\section{Modelling of exposure from different microenvironments is being used to explore the importance of different exposure sources and particle sizes}

$\mathrm{E}$ pidemiological studies have shown that the low levels of air pollution in most industrialised societies are still linked to adverse health outcomes. There is currently considerable research activity to investigate the possible associations between short term fluctuations in airborne particulate matter and morbidity or mortality, ${ }^{12}$ and mortality from long term chronic exposure. ${ }^{3}$ The World Health Organisation suggests that a 100 $\mu \mathrm{g} / \mathrm{m}^{3}$ increase in the daily average concentration of particulate matter, measured as $\mathrm{PM}_{10}$ (that is, particulate matter with average diameter less than $10 \mu \mathrm{m})$, could result in a $7 \%$ increase in daily mortality and an $8 \%$ increase in daily hospital admissions. The public health consequences of long term exposure to particulate matter may be substantially greater. If the associations are truly causal then they present important challenges for public health protection.

Most of the epidemiological investigations rely on measurements of the pollutant at some central location in the city and then use these data as a surrogate for individual exposure. For example, in a recent study of the daily mortality and hospital admissions in the West Midlands of the UK, the exposure of the 2.3 million people in the study area to particulate matter, carbon monoxide, nitrogen dioxide, and other pollutants was defined by data from between one and three monitoring stations. ${ }^{4}$ Although the daily variation in pollutant concentrations was relatively small (the 90th centile of the 24 hour average $\mathrm{PM}_{10}$ concentrations was only $38 \mu \mathrm{g} / \mathrm{m}^{3}$ ), the authors were still able to identify associations between respiratory and asthma admissions to hospital for young people and various measures of particulate concentration. Similar associations for mortality and hospital admissions from respiratory disease in elderly people were also identified.

In reality the exposure of the people at risk must come from a multiplicity of sources, inside buildings, in vehicles, and in the general urban environment. The population includes all ages, with some living an active lifestyle and others, who are perhaps chronically sick, being limited in what they can do. The possibility for differences between personal exposure and fixed point concentration measurements in such studies must therefore be great. It is to be expected that the type of "misclassification" that might arise in an epidemiological study because of this would tend to obscure any causal associations between exposure and ill health, which may partly explain the difficulties of some studies to identify associations. However, there is generally a consistency in the association between particulate matter measured at a central site and individual health, which some have argued is paradoxical, although it may be that the urban background concentrations better reflect the aerosol fraction that carries the risk.

Much of the recent epidemiology has been based on time-series studies of large urban populations, although there are also a number of small panel studies of groups that may be more susceptible to the effects air pollutants. Studies of the long term effects of air pollution have been carried out in large cohorts. In all of these investigations it would be possible to undertake some personal exposure monitoring to support the study interpretation, although clearly in the larger studies where there may be several hundreds of millions of person-days of observation it would be impractical to obtain sufficient data to directly estimate exposures. Unfortunately, the consequence of this is that generally no exposure measurements are made.

In panel studies we would ideally wish to measure the exposure of everyone every time a health observation was made or even on a representative number of occasions. However, there are practical difficulties in using personal pump based monitors to evaluate exposures over 24 hours. Even in major research studies it is exceptional to find more than 100 measurements of personal exposure to particulate air pollutants. Some progress is being made to develop smaller, lighter samplers to collect personal measurements, but until such instruments become available the solution must be to develop reliable strategies to estimate exposures in the absence of measurements so that these techniques can be used in epidemiological studies.

In this issue, Harrison and his coworkers ${ }^{5}$ investigate the relation between personal exposure and concentration measurements made in a number of "microenvironments" that the subjects pass through. The aim of the research was to identify whether it is possible to produce reliable estimates of exposure using modelling, based on time-activity diaries kept by the subjects. The study looked at three pollutants: carbon monoxide, nitrogen dioxide, and particulate matter (as $\left.\mathrm{PM}_{10}\right)$. The authors found good associations between personal exposure and microenvironment concentrations for each of the pollutants, although their modelled exposures were unable to encompass the whole range found in measurements. Nevertheless, their work makes an important contribution to developing such techniques for use in epidemiological studies.

Particulate matter is a heterogeneous mixture of many different substances which may range from a few nanometres in size up to tens of micrometres. The available toxicological information would suggest that it is the very small particles that may be most important in producing adverse health effects. In fact there are some suggestions that although people spend most of their life indoors, it is outdoor combustion related sources that are the cause of the observed morbidity and mortality. This could explain why using data from city centre monitoring stations has consistently produced associations with morbidity and mortality.

Harrison and his coworkers also highlight an important difference between particulate exposure measurements and the gaseous contaminants, what they call a "personal cloud" effect. This personal cloud appears to be a fairly constant background level of exposure associated with the individual, perhaps because they resuspend dust from surfaces as they move about, although this is not fully understood. These relatively large resuspended particles $(>10 \mu \mathrm{m})$ may present no risk to health. In contrast some cooking activities can generate very high numbers of nanometre size particles that the toxicological studies would suggest should be more important for health. However, without some way of incorporating estimates of exposure from the various particle sources into epidemiological studies it will be difficult to investigate such issues. There are a number of panel studies underway, including a study of people with chronic lung disease in Aberdeen, where modelling of exposure from different microenvironments is 
being used to explore the importance of different exposure sources and particle sizes. Such studies offer a new opportunity to understand what aspects of particulate matter may cause ill health.

Occup Environ Med 2002;59:653-654

\section{Author's affiliation}

J W Cherrie, University of Aberdeen and Institute of Occupational Medicine
Correspondence to: J W Cherrie, University of Aberdeen and Institute of Occupational Medicine, 8 Roxburgh Place, Edinburgh

EH8 9SU, UK; john.cherrie@abdn.ac.uk

\section{REFERENCES}

Zanobetti A, Schwartz J, Dockery DW Airborne particles are a risk factor for hospital admissions for heart and lung disease. Environ Health Perspect 2000;108:1071107.

2 Schwartz J, Ballester F, Saez M, Pérez-Hoyos $\mathrm{S}$, et al. The concentration-response relation between air pollution and daily deaths. Environ Health Perspect 2001;109:1001-6.
3 Pope CA, Burnette RT, Thun M, et al. Lung cancer, cardiopulmonary mortality, and long-term exposure to fine particulate a pollution. JAMA 2002;287: 1132-41.

4 Anderson HR, Bremner SA, Atkinson RW, et al. Particulate matter and daily mortality and hospital admissions in the west midlands conurbation of the United Kingdom: associations with fine and coarse particles, black smok and Med 2001;58:504-10.

5 Harrison RM, Thornton CA, Lawrence RG, et al. Personal exposure monitoring of particulate matter, nitrogen dioxide and carbon monoxide, including susceptible groups. Occup Environ Med 2002;59: groups.

\section{Answers to multiple choice questions on Mental ill health and fitness for work by} N Glozier, on pages 714-720

(1) (a) true; (b) false; (c) false; (d) false; (e) true

(2) (a) false; (b) true; (c) true; (d) false; (e) true

(3) (a) false; (b) true; (c) true; (d) true; (e) false

(4) (a) true; (b) false; (c) false (d) false; (e) true 